\title{
Review of Online Teaching Platforms in the Current Period of COVID-19 Pandemic
}

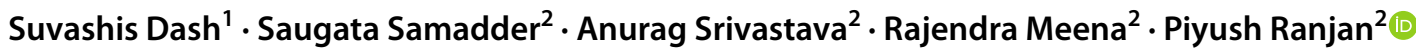

Received: 26 April 2021 / Accepted: 19 May 2021 /Published online: 18 June 2021

(c) Association of Surgeons of India 2021

\begin{abstract}
Online teaching platforms have become a core appealing option for education and information delivery in current pandemic of Coronavirus disease 2019 (COVID-19) among medical professionals. This editorial aims to understand perspective of usability and practicality of audio and video conferencing platforms in the current situation. Review of various available online platforms was done, namely Zoom, Google Meet, Google Classroom, Microsoft Teams, Cisco Webex, Go ToMeet, and Say Namaste highlighting and comparing their essential features, their benefits, the system and operating system in which they are supported, user interface, number of individuals who can participate, price packages, security, customer support, and limitations. Based on this, educational implications are discussed and are a guide to choose a suitable platform as well as suggestion for future research.
\end{abstract}

Keywords Online teaching platform $\cdot$ Audio and video conferencing platforms $\cdot$ Education $\cdot$ Information delivery

\section{Introduction}

Online education is becoming the attractive mode of learning for many of us in the current Coronavirus disease 2019 (COVID-19) pandemic period. Sometime ago, the digital India movement was initiated and intended to transform every avenue of our society with hassle-free use of digital technology. This current situation presents such an opportunity among adversity to use digital technology. In India, there are more than 480 million internet users (2018 data), and India is presently ranked as the second largest online

Piyush Ranjan

drpmishra28@gmail.com

Suvashis Dash

suvashis.dash@gmail.com

Saugata Samadder

samaddersaugata.ss@gmail.com

Anurag Srivastava

dr.anuragsrivastava@gmail.com

Rajendra Meena

rajaiims2734@gmail.com

1 Department of Plastic, Reconstructive and Burn Surgery, All India Institute of Medical Sciences, New Delhi, India

2 Department of Surgical Disciplines, All India Institute of Medical Sciences, New Delhi, India consumer in world. By year 2021, it will be predicted to cross 660 million (https://www.statista.com/statistics/ 255146/number-of-internet-users-in-india/). Like other sectors, the healthcare sector has also ingrained the use of information technology for various services, from better delivery of healthcare services and for continuum of learning and research. This brings a change in the traditional paradigm of education delivery, the shift from scribbling of pen and paper classroom to digital "log in" to the virtual classroom. It is found that around $80 \%$ of doctors use internet for professional purpose and to enhance their knowledge (https:// www.ipsos.com/en-ru/74-doctors-use-internet-profession al-purposes).

The traditional classroom teaching is a widely accepted and time-tested mode. The physical presence, the verbal and nonverbals cues, and a wholesome experience of the traditional classroom are unmatched. Many will accept that the attributes of a collaborative, organized, disciplined, stimulating good classroom teaching cannot be replaced by the online teaching in the near future; however, to circumvent these difficult times, where the meetings and mass gatherings are forbidden and social distancing is the norm, the digital conferencing platforms to deliver the required education in the form of webinars are the new normal. Although these platforms are trying to incorporate the latest technology to elude the malady of digital teaching, they still need 
to traverse a lot of advancement and refinement essential for acceptability in times to come. Moreover, another aspect to ponder is the rural and urban distribution; can online classes be successful in rural or semi-urban areas? The latest statistics shows that there is a high growth in the urban and rural areas, with demand for mobile-based internet technology (https://www.statista.com/statistics/255146/number-of-inter net-users-in-india/).

Global surveys show that only 2 countries are comfortable sending their children back to school (https://www. ipsos.com/en-ru/more-people-want-return-work-uncomforta ble-kids-returning-school-amid-covid-19), while most of the respondents are not comfortable sending their children to school, in the near future owing to COVID-19 scare. This scenario prevails in the United States of America (USA), the United Kingdom (UK), Russia, Italy, Spain, Germany, China, and India where $92 \%$ parents are unwilling to send their children to school (https://economictimes.indiatimes. com/magazines/panache/92-indian-parents-unwilling-tosend-their-child-back-to-school-immediately-upon-reope ning/articleshow/75712766.cms?from =mdr).

Although some consider the internet speed and availability of network as an issue for online education, the situation is changing gradually in the last 5 years. However, during lockdown period, due to heavy consumption of internet traffic, there is some drop in the speed (https://www.india times.com/technology/news/indias-internet-speed-is-falli ng-since-jan-2020-globally-rank-130th-for-mobile-dataspeed-510505.html).

We provide some insight in this article regarding the attributes of popular digital conferencing platforms used among medical professionals and their usability and practicality in the current situation (Fig. 1, Table 1).

\section{Zoom}

It is a preferred tool for many to make videoconference due to its well-balanced features. The intuitive user interface, integration with "Outlook," is simple and intuitive; one-click meetings can be initiated from desktops, mobiles, and tablets. It is easy and quick to install. The pricing and license options are publicly available; cost structure is transparent and easy to understand.

Zoom webinar allows to host events with up to 100 interactive participants through high-definition audio and video conference and 1000 view-only attendees with a free account. One can also request for detailed analytics about the number of meetings that have taken place, how many members have attended on an average, how much time does an average session last for, and the likes.

The "Zoom Pro" application is the advanced version in which one can raise the number of participants to 500 and even 1000, for a one-time fee of \$64.99 (Rs 4961) and $\$ 104.99$ (Rs 8015) respectively. It is available on various operating systems (OS) like windows, Linux, macOS X, iOS, Android, and BlackBerry.

Freedom like generating a unique meeting identity (ID) which is more convenient for small group meeting regularly, recording a meeting locally or cloud storage (paid plans) so it can be shared with people who could not attend, a choice for everyone to enable or disable their video at any time, chat rooms for group or individual conversations, dual screen support, ability to link to calendar application like Google Calendar and Outlook are some of its features. Live broadcasts via social channels and YouTube, host controls like mute/unmute use of white board tool to express the thoughts to the attendees, and more make it a premier application.
Fig. 1 Features of virtual conferencing platforms required for online learning

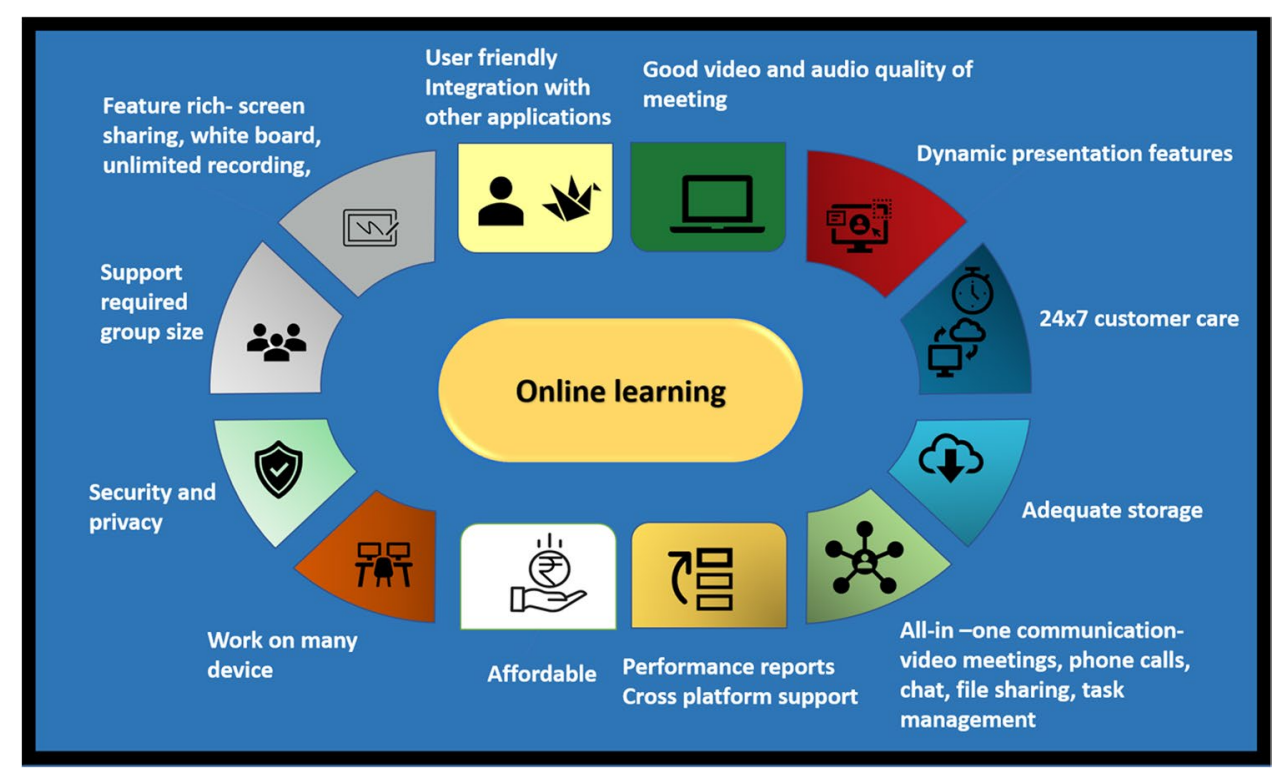




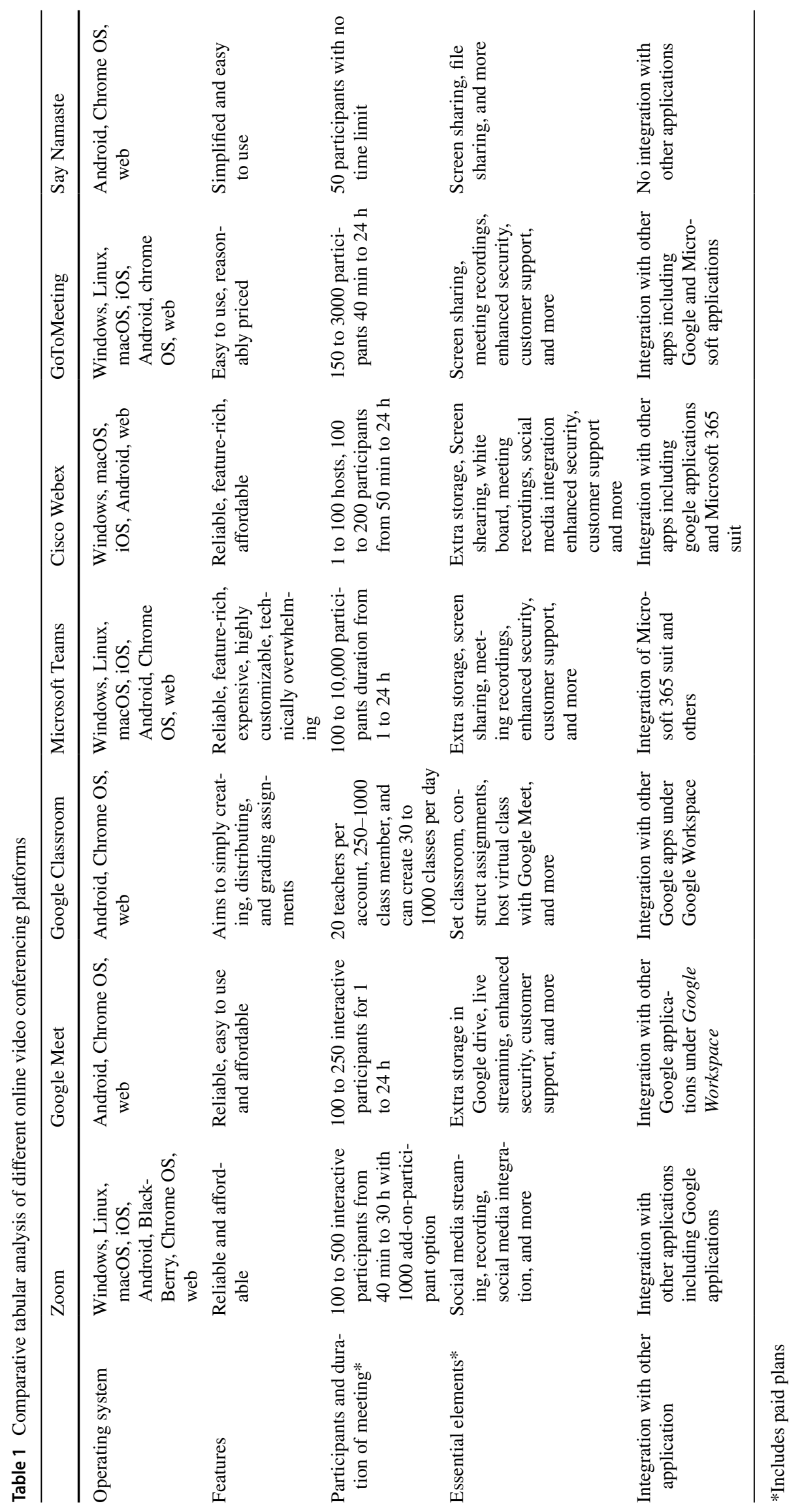


Everything is not jolly about Zoom; certain setbacks might disappoint the user like varying degrees of lag, particularly with the audio. There can be an issue in syncing across devices or accounts so it might end up sending new invites to the same people for the same meeting, therefore causing more confusion.

Zoom has become more popular as people get to videocalling applications amid the ongoing coronavirus pandemic. As more and more people use it, it is facing huge privacy and security backlash as experts caution default settings are not secure enough.

Settings differ between the locally installed application and the web application, and the settings are plentiful and worth revisiting from time to time.

\section{Google Meet}

Google Meet is a popular video conferencing platform because of its accessibility, affordability, excellent inbuilt video conferencing tools, and robust security features. It is available on Android, iOS, and Chrome, and it is easy to set up virtual conferences for up to 250 people.

Being a part of $\mathrm{G}$ suit, it offers three professional plans — basic-Rs. 125 (\$1.72), business-Rs. 672 (\$9.26), and enterprise-Rs. 1650 (\$22.73) per month per person respectively thus allowing to take full advantages of the additional programs that come with it like drive, slide presentation builder, Google docs, spreadsheet, Cloud search, and more of which most of us carry in our devices.

The interface is clean and has clearly labeled commands that make it easy to get a conversation started especially for those who do frequent ad hoc virtual communications. While it may not provide an elaborate tool that other video conferencing platforms are offering, it provides some of the most valuable tools like "host can share their screen," "digital presentations," documents, spreadsheet, or other files they want with the attendees. Participants can enter the meet directly from the Google calendar, once an event is created, on that particular date with the meeting link. Camera and audio on/off button do exist along with displaying who is in the meeting and harbor a chat room with a range of features like live polling to make decisions. Enterprise plan allows automatically "save the video" in Google drive and "live stream viewing" for up to 100,000 viewers.

The setback is that it can be accessed only by Google Chrome. The mobile version is superior to the desktop version, but lacks services like white board tool. Lastly, more focus on Google products makes its application limited and might create delay for those unfamiliar to it.

\section{Google Classroom}

Google Classroom is part of "Google For Education" targeted to teachers and students in higher-education markets. Since 2018, it is available as an additional service in G Suit basic, business, and enterprise packages. The biggest plus is it is simple to use and is for everyone. It encourages collaboration between students and teachers which involves creating a class and add contents such as assignments, video, images, portable document format (PDF)s, Google Docs, or Google forms in a few clicks and then add students by name or send them link to join. It allows the host to manage a classroom and gives control which group can use the classroom and which cannot. Being a platform in the Google ecosystem, it uses the same Google apps that we are already familiar using in our mobile devices. The teacher needs to build online classes or homework on their own as well as can create additional classes which are shared with the students which they can view or download. The teacher can track and access each student's performance followed by effective feedback.

A limitation of Google Classroom includes the need of a separate account (other than the Gmail account) and might be frustrating to $\log$ in and $\log$ out in different accounts for sharing and accessing files and documents. As evident of being too googlish, things are easy as long as tools are aligned to Google services, if not then it becomes difficult and exhaustive. In the absence of any automated update, it needs frequent refreshes to get any update so that no important announcement is missed. The learners cannot share the work with their peers, until they become owners of that particular document, and once they become owners of the document, only then they can edit and modify the assignment. The major drawback of Google Classroom is not including Hangouts, making no online interaction or discussion possible in the platform.

\section{Microsoft Teams}

Microsoft Teams provides a platform that enables the users to instantaneously switch between messaging and video conferencing, and the benefits of native Mac. It is available on Android, iOS, and Chrome. One can schedule videos or audio meetings with a single person or team and can also organize webinars and large meetings up to 10,000 participants. With a free account, 299 people can be invited and gives all the primary features but lacks integration with office applications such as schedule meetings, share point access, security support, and compliance tools. There are three versions, business essential for United States Dollar 
(USD) $5 \$$ per person per month, business premium for USD \$12.50 per person per month, and enterprise for USD \$20 per person per month with annual commitment for all thus making it one of the most expensive video conferencing options. Microsoft Teams also provides the full range of features expected from a leading video conferencing software provider, including desktop sharing so the participants can also interact at the same time on the same page as you are, and the application-sharing option which allows third party or native programs to enter and share in the meeting and also call recording, live captions, background blur technology, and chat functionalities with clear audio and video, and quick multimedia files uploads. It also allows anonymous participants to enter the meeting other than the group members with one click, and they can interact using voice call or chats. Microsoft also offers analytical tools to review and improve the quality of the meets for the administrator.

The desktop app or web app is easier to use than the mobile version. Navigating tools might be confusing. As it is a Microsoft product, the only groups who will ever use Microsoft Teams to full potential are those who have heavily invested in Microsoft work place.

\section{Cisco Webex}

Webex Meetings has both desktop and mobile apps plus a web app that gives you control of the conference from your web browser. The biggest benefit is that you essentially get two platforms in one: On one side, you have a full video conference service that allows you to host video conferences with large numbers of participants inside and outside your organization through Webex Meetings. On the other side, you have Webex Teams, which provides you with a platform designed specifically to allow your staff to connect with each other. It allows to share ideas through messaging, video meetings, and white boarding. With its free service, it allows 100 participants at a time and does not limit the duration of the meeting as compared to others which have limits on the number of participants and how long the meeting can last.

Between Webex Meetings and Webex Teams, there are several features that businesses of all sizes will find valuable. Webex Meetings gives full control over conference over both desktop and mobile apps plus web apps. Meeting serves as a conference call where those invited might not be able to see but can share thoughts using a dial-in number. It also integrates with popular programs including Microsoft Office 365, Outlook, and Google Calendar. Webex Team has its own set of features like messaging, file sharing, and white boarding. One of the best features is audio-to-text transcripts that Webex creates for each meeting. So, one can choose to automatically receive recording transcriptions and do not have to focus on making notes during your meeting.

The free version comes with desktop-sharing, application-sharing, file-sharing, and whiteboard-sharing options; high-definition (HD) video, active speaker, thumbnail, grid, and floating views; personal rooms; web apps, calendar integrations, instant and scheduled meetings, host meeting controls, question and answer, polling and raise-hand features, interactive whiteboards, unlimited messaging and file sharing, emoji and Graphics Interchange Format (GIF) sharing, mobile screen sharing, and mobile voice command integrations with meeting participants up to 100 .

The starter plan at USD \$14.95 per host per month comes with cloud storage of 5 gigabytes, everything in the free plan, plus recording transcriptions, Motion Picture Experts Group-4 Part 14 (MP4) meeting recordings, customizable personal room links, the ability to join from a video system or application, user management and administrative controls for 50 participants, Plus plan at USD \$19.95, and Business plan at USD \$29.95 per host per month with some further advanced upgrades and limits up to 100 and 200 participants respectively.

Technical issues, cost, and number of participants are the same as Webex. No doubt being functional, feature-rich, and reliably secure, Cisco Webex is one of the best alternatives to the abovementioned conferencing platforms.

\section{GoToMeeting}

It is one of the popular online meeting applications. It has many features, even with the free plan, it offers a meeting. GoToMeeting is a simple platform and has an easy user interface. By one click/press, the user can start the meeting. It allows the users to start their meetings by variety of platforms or applications and can share their screen. The user can also start the meeting from their email links or chat box. In the free plan, the video conference is limited to only 3 participants which is one of its limitations.

\section{Say Namaste}

This is an Indian app, developed by an Indian startup. It was earlier available only in web version, which is now available for smartphones. The application is easy to use and have good user experience. For video conferencing, one meeting room is created. For starting a meeting, a code needs to be created and can be shared via email or chat box. By clicking the link, one can join the video conference. This application supports up to 50 participants in each conference. Features such as screen sharing, file sharing, and chatting are available in this application. Similar to Zoom and other 
video-calling platforms, the screen sharing option on Say Namaste lets users easily share screen using desktop/laptop, and the shared screen can be used by all participants in the app itself. This application creators provide regular updates to fix the problems and glitches. It is very popular with good rating presently.

\section{Conclusion}

The type of health communication being used like a demonstration which is a carefully prepared presentation to show how to perform a skill or procedure can be carried out using a previously made video and describing it or demonstrating live. Group discussion is a very effective method which has at max 12 members can easily conduct conference in the abovementioned platform. Panel discussion which includes 4 to 8 people qualified to talk about the topic and discussion on a given problem can be conducted in front of large audience virtually by keeping the majors in the group and the others as invitee. Symposia, conference, and seminars which range from half day to 1 week in duration with ideally as small as 7-15 participants in seminar to about 75 participants in conference can be preplanned using calendar application and sending invites through emails. Conducting workshop which has group of 4 or more involving consultants and resource panel can also focus on individual work under a friendly, happy, and democratic atmosphere through this online group meet.

The number of similar platforms to boost this online teaching is numerous, and the features each program can provide are limitless, but the answer to a perfect video conferencing service is none. Zoom suits all kinds of health communication methods which includes a large number of participants. If one wants to invest for more added features to the same with enhanced security and customer support, one can opt for Cisco Webex and Microsoft Teams. If one needs fast interface and to reduce wait or have small groups like workshop, one can go for Google Meet or GoToMeeting. Assignments and classes for academic purpose for undergraduates or postgraduates can be done smoothly through Google Classroom and Google Meet under the umbrella of Google Workspace. The choice depends on so many factors like distribution and uniformity of technology advancement of the users, computer literacy, ability of the administrator to organize and incorporate, and ability of the user to retrieve the resources, and detailed ground work.

Data Availability Not applicable.

Code Availability Not applicable.

\section{Declarations}

Ethics Approval Not applicable.

Consent to Participate Not applicable.

Consent for Publication Not applicable.

Competing Interests The authors declare no competing interests.

Publisher's Note Springer Nature remains neutral with regard to jurisdictional claims in published maps and institutional affiliations. 